Handbook for Venous Thromboembolism 

Gregory Piazza • Benjamin Hohlfelder Samuel Z. Goldhaber

\section{Handbook for Venous Thromboembolism}

望 Springer 
Gregory Piazza

Cardiovascular Division

Harvard Medical School

Brigham and Women's Hospital

Boston, Massachusetts

USA

Benjamin Hohlfelder

Department of Pharmacy Services

Brigham and Women's Hospital

Boston, Massachusetts

USA
Samuel Z. Goldhaber

Thrombosis Research Group

Harvard Medical School

Brigham and Women's Hospital

Boston, Massachusetts

USA

ISBN 978-3-319-20842-8

ISBN 978-3-319-20843-5 (eBook)

DOI 10.1007/978-3-319-20843-5

Library of Congress Control Number: 2015948376

Springer Cham Heidelberg New York Dordrecht London

(C) Springer International Publishing Switzerland 2015

This work is subject to copyright. All rights are reserved by the Publisher, whether the whole or part of the material is concerned, specifically the rights of translation, reprinting, reuse of illustrations, recitation, broadcasting, reproduction on microfilms or in any other physical way, and transmission or information storage and retrieval, electronic adaptation, computer software, or by similar or dissimilar methodology now known or hereafter developed.

The use of general descriptive names, registered names, trademarks, service marks, etc. in this publication does not imply, even in the absence of a specific statement, that such names are exempt from the relevant protective laws and regulations and therefore free for general use.

The publisher, the authors and the editors are safe to assume that the advice and information in this book are believed to be true and accurate at the date of publication. Neither the publisher nor the authors or the editors give a warranty, express or implied, with respect to the material contained herein or for any errors or omissions that may have been made.

Printed on acid-free paper

Springer International Publishing AG Switzerland is part of Springer Science+Business Media (www.springer.com) 
The authors dedicate this book to their mentors, colleagues, and families for their inspiration, guidance, and support. 



\section{Preface}

Venous thromboembolism (VTE), including deep vein thrombosis (DVT) and pulmonary embolism (PE), is the third most common cardiovascular disorder after myocardial infarction (MI) and stroke. Unlike MI and stroke, which occur most commonly in older patients with numerous medical comorbid conditions, VTE afflicts both the young and old and the seemingly healthy and chronically infirmed. Diagnosis and management of VTE cross the boundaries of many disciplines of medicine. PE and DVT are common complications of major surgery, trauma, cancer, and hospitalization for medical illness. VTE is also an important women's health concern, especially during and after pregnancy and with use of hormonal contraceptive or replacement therapy. Accordingly, an understanding of the risk factors, pathophysiology, diagnosis, treatment, and prevention of VTE is critical for most clinicians, not only cardiovascular medicine specialists.

Over the past three decades, the Brigham and Women's Hospital (BWH) Thrombosis Research Group has focused on advancing patient care and clinical investigation in the field of VTE. From epidemiological studies that have promoted our greater understanding of risk factors to pivotal trials of fibrinolytic therapy for treatment of acute PE and computerized decision support to prevent VTE in highrisk patients, the BWH Thrombosis Research Group has sought to bring the breakthroughs in clinical research to the bedside. More than a decade ago, we were approached by our Healthcare System leadership and asked to draft a set of evidence-based clinical practice guidelines that would help our clinicians navigate the rapid advances in VTE diagnosis, management, and prevention. In response, we drafted the first edition of the Venous Thromboembolism Guidebook.

Because the field of VTE has evolved, we have published online and in print five subsequent editions of the Venous Thromboembolism Guidebook. The emergence of the non-vitamin $\mathrm{K}$ oral anticoagulants and new catheter-based techniques for treatment of DVT and PE have presented an ongoing challenge to clinicians at our medical center, throughout the USA, and worldwide. We are delighted to bring our decades of experience and expertise in evaluation and treatment of patients with VTE to a wider audience in the form of this Handbook of Venous Thromboembolism. 
In the Handbook, we provide a state-of-the-art but practical guide to understanding the risk factors and pathophysiology, diagnosis and risk stratification, and treatment and prevention of VTE. We use clinical vignettes and self-assessment questions to emphasize key concepts from each chapter. We hope that this Handbook of Venous Thromboembolism will enable readers to incorporate changes in our rapidly advancing field into the daily care of patients who have suffered or are at risk for developing VTE.

Boston, MA, USA

Gregory Piazza, MD, MS 


\section{Contents}

1 Overview and Epidemiology of Venous Thromboembolism . . . . . . . . 1

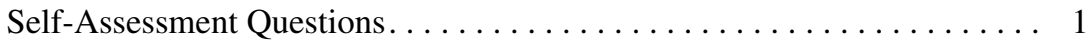

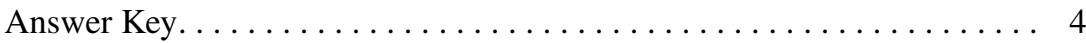

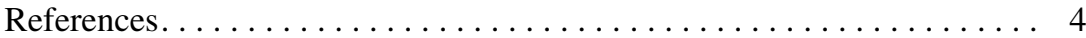

2 Risk Factors for Venous Thromboembolism: Recognizing the Spectrum of Risk and Understanding the Role

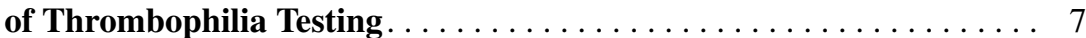

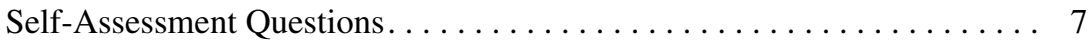

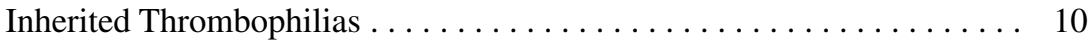

Lifestyle-Related Risk Factors $\ldots \ldots \ldots \ldots \ldots \ldots \ldots \ldots \ldots \ldots \ldots$

Acquired Risk Factors. . . . . . . . . . . . . . . . . . . . . . 10

Thrombophilia Evaluation $\ldots \ldots \ldots \ldots \ldots \ldots \ldots \ldots \ldots \ldots \ldots \ldots$

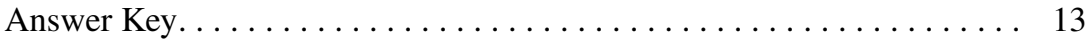

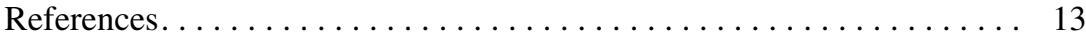

3 Pathophysiology of Deep Vein Thrombosis

and Pulmonary Embolism: Beyond Virchow's Triad . . . . . . . . 15

Self-Assessment Questions. . . . . . . . . . . . . . . . . . . 15

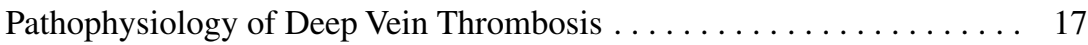

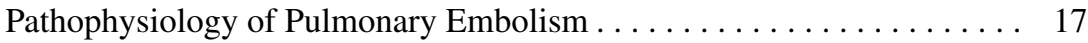

Answer Key. . . . . . . . . . . . . . . . . . . . . . . . . . . . . . . 19

References........................ 20

4 Diagnosis of Deep Vein Thrombosis: Incorporating Clinical

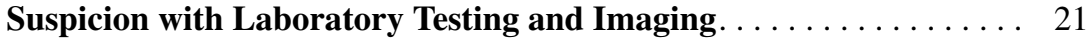

Self-Assessment Questions. . . . . . . . . . . . . . . . . 21

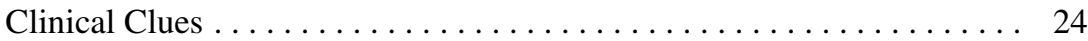

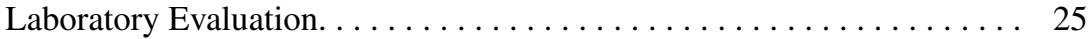

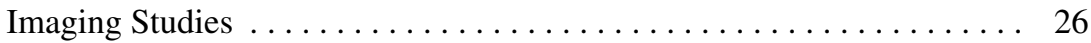

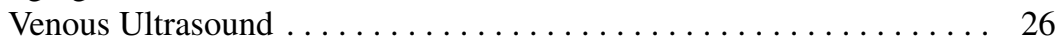

Other Imaging Modalities . . . . . . . . . . . . . . . . 26 


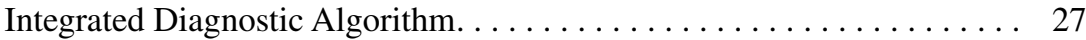

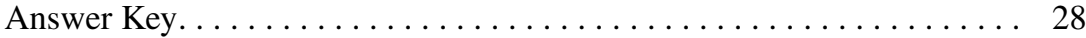

References................................ 28

5 Diagnosis of Pulmonary Embolism: An Integrated Approach to Clinical Evaluation, Laboratory Testing, and Imaging . . . . . . . . 29

Self-Assessment Questions.......................... 29

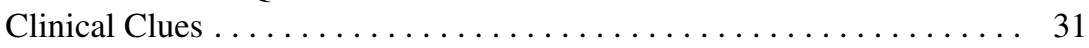

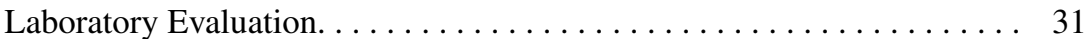

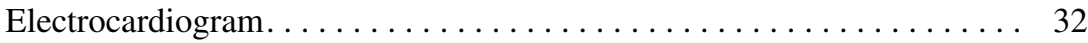

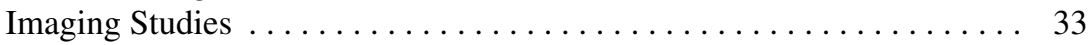

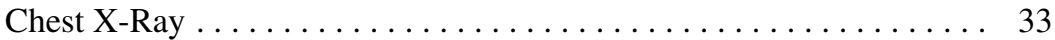

Chest Computed Tomography. . . . . . . . . . . . . . . . . . . 33

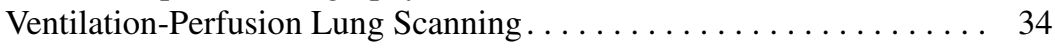

Magnetic Resonance Angiography . . . . . . . . . . . . . . . 34

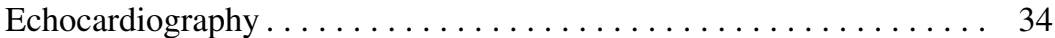

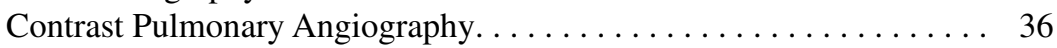

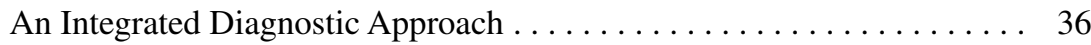

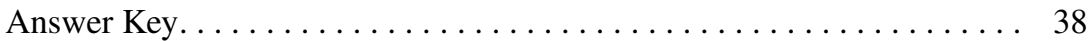

References.................................. 39

6 Risk Stratification and Prognosis: Identifying Patients

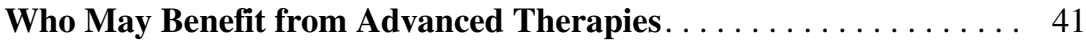

Self-Assessment Questions. .................... 41

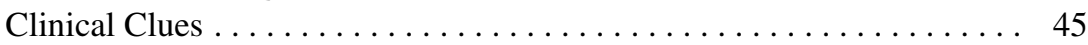

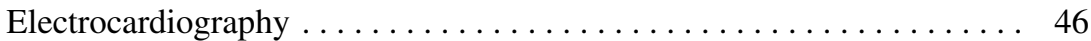

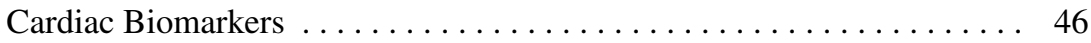

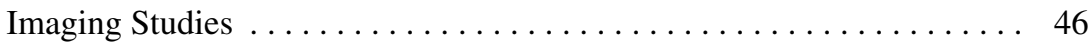

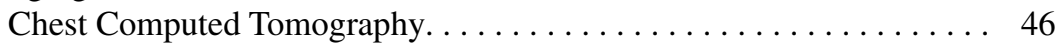

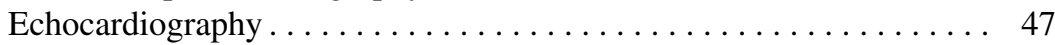

An Integrated Approach to Risk Stratification . . . . . . . . . . . 47

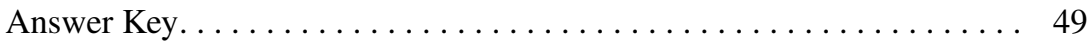

References............................... 49

7 Advanced Therapy for Venous Thromboembolism:

Understanding the Role of Systemic Fibrinolysis,

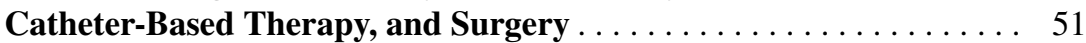

Self-Assessment Questions...................... 51

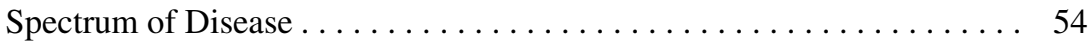

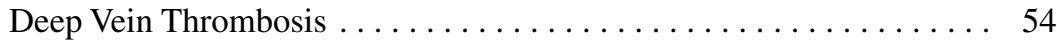

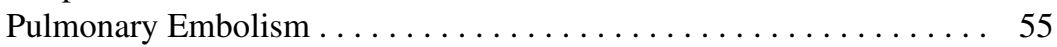

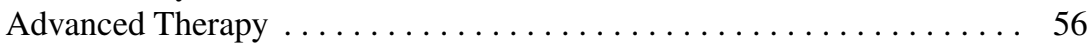

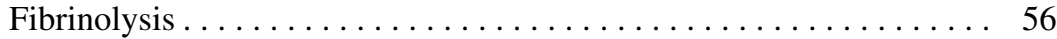

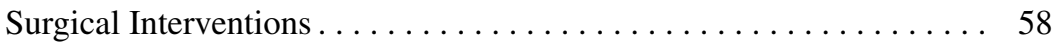

Catheter-Assisted Pharmacomechanical Therapy. . . . . . . . . . 59

Adjunctive Therapy for Patients with Massive Pulmonary Embolism. . . . 62 
Extracorporeal Membrane Oxygenation. ............... 63

Pulmonary Embolism Response Teams . . . . . . . . . . . . . 63

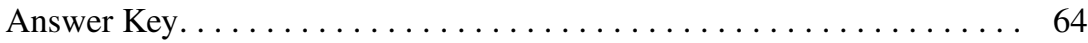

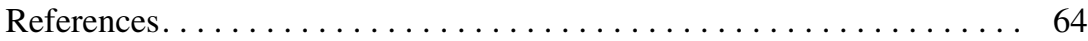

8 Inferior Vena Cava Filters: Recognizing Indications

for Placement and Retrieval. . . . . . . . . . . . . . . . . . . . . 67

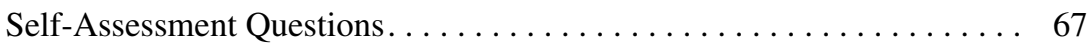

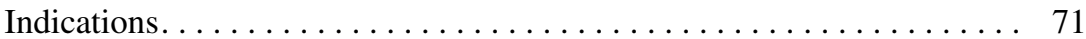

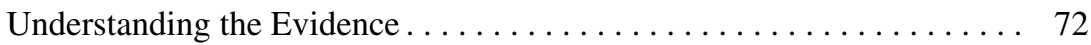

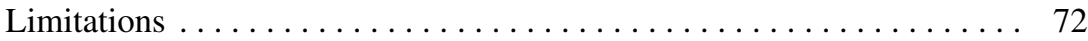

Practical Considerations . . . . . . . . . . . . . . . . . . 72

Answer Key. . . . . . . . . . . . . . . . . . . . . . . 75

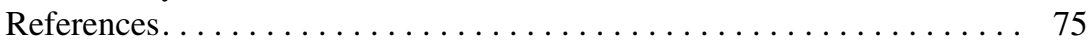

9 Anticoagulation for Venous Thromboembolism:

Selecting the Optimal Parenteral and Oral

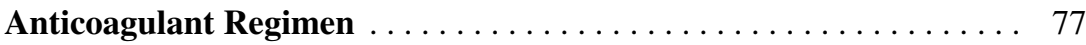

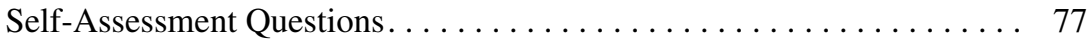

Parenteral Anticoagulation . . . . . . . . . . . . . . . . . . . . 80

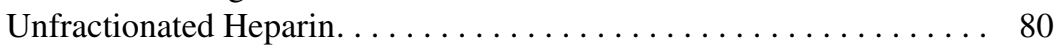

Low-Molecular Weight Heparin . . . . . . . . . . . . . . . . . 80

Adverse Effects of Heparin. . . . . . . . . . . . . . . . . . . 81

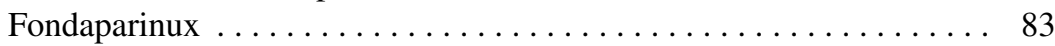

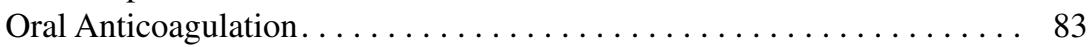

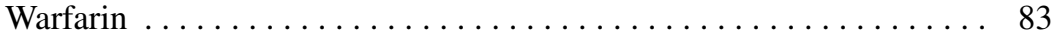

Non-vitamin K Oral Anticoagulants (NOACs) . . . . . . . . . . . . 84

Management of Oral Anticoagulant Associated-Bleeding . . . . . . . . 87

Tips for Outpatient Anticoagulation . . . . . . . . . . . . . . 87

Answer Key. . . . . . . . . . . . . . . . . . . . . . . . . . . 89

References........................... 89

10 Long-Term Management of Venous Thromboembolism:

Strategies for Reducing the Risk of Recurrence . . . . . . . . . . . 93

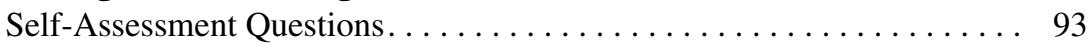

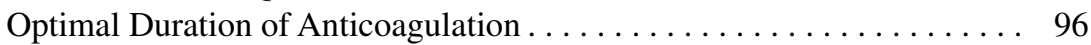

Aspirin for Prevention of Recurrent Venous Thromboembolism . . . . . . . 97

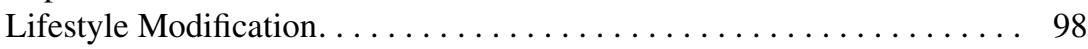

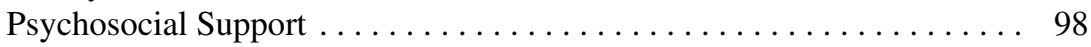

Follow-Up After Venous Thromboembolism . . . . . . . . . . . . . . . 98

Answer Key. . . . . . . . . . . . . . . . . . . . . . . . . . 99

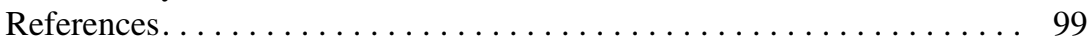

11 Chronic Thromboembolic Pulmonary Hypertension:

A Pathophysiologic Basis for Diagnosis and Management . . . . . . . 101

Self-Assessment Questions. . . . . . . . . . . . . . . . . . . 101

Epidemiology ................................ 104 


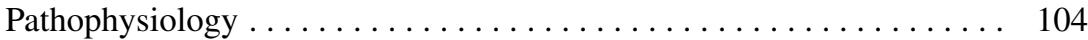

Clinical Presentation . . . . . . . . . . . . . . . . . . . . . . . . . . . 104

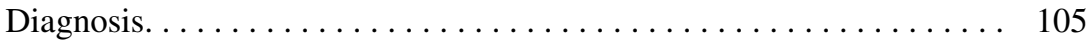

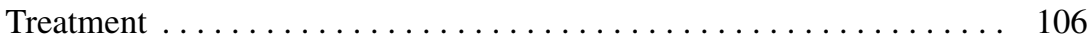

Pulmonary Thromboendarterectomy . . . . . . . . . . . . . 106

Balloon Pulmonary Artery Angioplasty . . . . . . . . . . . . . . . . 107

Medical Therapy . . . . . . . . . . . . . . . . . . . . . 107

Overall Therapeutic Algorithm . . . . . . . . . . . . . . . . . . 108

Answer Key. . . . . . . . . . . . . . . . . . . . . . . . . . . . . . . 109

References.............................. 109

12 Post-thrombotic Syndrome: Recognizing and Treating a Debilitating Complication of Deep Vein Thrombosis . . . . . . . . . 111

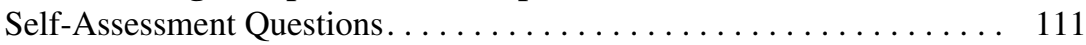

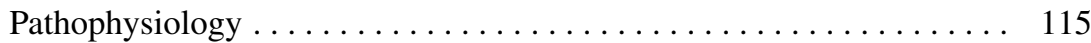

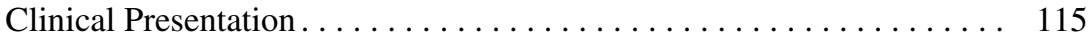

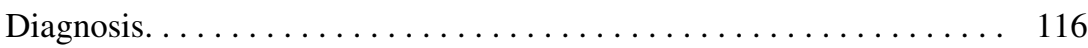

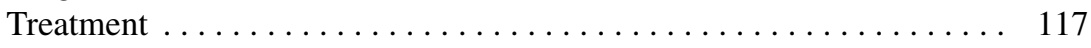

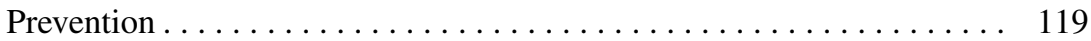

Answer Key. . . . . . . . . . . . . . . . . . . . . . . 120

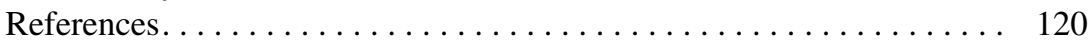

13 Prevention of Venous Thromboembolism:

An Evidence-Based Approach to Thromboprophylaxis . . . . . . . . . 123

Self-Assessment Questions. . . . . . . . . . . . . . . . . . 123

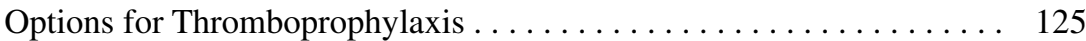

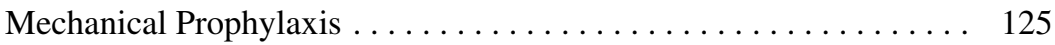

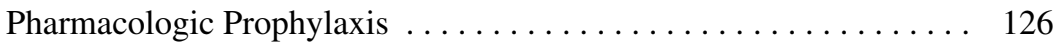

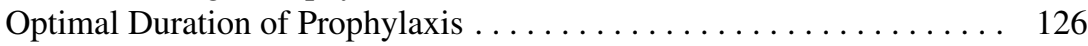

Recommendations for Specific Patient Populations . . . . . . . . . . . . 127

Medical Inpatients. . . . . . . . . . . . . . . . . . . . 127

Medical Patients After Discharge . . . . . . . . . . . . . . . . . 127

Orthopedic Patients. . . . . . . . . . . . . . . . . . . . . . . . 128

Abdominal or Pelvic Surgery for Malignancy . . . . . . . . . . . . . . . . 129

Medical Oncology Patients . . . . . . . . . . . . . . . . . . . . . . . 129

An Overview of Prophylactic Regimens. . . . . . . . . . . . . . . 130

Answer Key. . . . . . . . . . . . . . . . . . . . . . . . . 130

References............................ 131

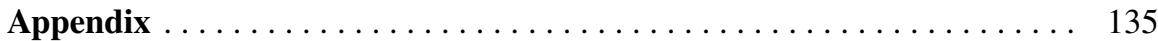

Index. . . . . . . . . . . . . . . . . . . . . . . . . . . . 139 


\section{Abbreviations}

$\begin{array}{ll}\text { aPTT } & \text { Activated partial thromboplastin time } \\ \text { CT } & \text { Computed tomogram } \\ \text { DVT } & \text { Deep vein thrombosis } \\ \text { INR } & \text { International normalized ratio } \\ \text { IV } & \text { Intravenous } \\ \text { IVC } & \text { Inferior vena cava } \\ \text { LMWH } & \text { Low molecular weight heparin } \\ \text { LV } & \text { Left ventricle } \\ \text { MR } & \text { Magnetic resonance } \\ \text { NOAC } & \text { Non-vitamin K oral anticoagulant } \\ \text { PCC } & \text { Prothrombin-complex concentrate } \\ \text { PE } & \text { Pulmonary embolism } \\ \text { PTS } & \text { Post-thrombotic syndrome } \\ \text { RV } & \text { Right ventricle } \\ \text { SC } & \text { Subcutaneous } \\ \text { VTE } & \text { Venous thromboembolism }\end{array}$

